\title{
A Diagnosis Missed for Several Years- Wegener's Granulomatosis
}

\author{
Paudyal BP, ${ }^{1}$ Pantha $S,{ }^{1}$ Ranjitkar $N,{ }^{1}$ Manandhar $A,{ }^{2}$ Arjyal $\mathrm{A}^{3}$
}

${ }^{1}$ Department of Medicine

${ }^{3}$ Department of Community Health Sciences

Patan Academy of Health Sciences

Patan Hospital, Lalitpur, Nepal

${ }^{2}$ Tilganga Institute of Ophthalmology

National Academy of Medical Sciences

Kathmandu, Nepal

\section{CORRESPONDING}

Buddhi Paudyal

Department of Medicine

Patan Academy of Health Sciences

Patan Hospital, Lalitpur, Nepal.

E-mail: buddhipaudyal@yahoo.com

Citation

Paudyal BP, Pantha S, Ranjitkar N, Manandhar A, Arjyal A. A diagnosis missed for several years -

Wegener's granulomatosis. Kathmandu Univ Med J 2011;35(3):218-21.

\begin{abstract}
Wegener's granulomatosis is a form of systemic vasculitis of small to medium sized vessels and affects upper respiratory tract, lungs and kidneys along with various organs. It causes necrotizing granulomatous inflammation of the affected parts and presents with positive antineutrophil cytoplasmic antibodies in more severe forms. Being a systemic disease with the potential to affect any organ-systems with a wide range of clinical presentations, it is associated with a risk of delay in diagnosis with resultant setback in institution of appropriate treatment. Confusion may arise due to an extent of histological similarity between Wegener's granulomatosis and the more prevalent tuberculosis, both causing granulomatous inflammation of the affected parts. Here, we present two cases of this rare disorder where the diagnosis was missed for several years in the beginning causing a delay in institution of specific therapy which led to the development of complications.
\end{abstract}

\section{KEY WORDS}

delayed diagnosis, multisystem disease, systemic vasculitis, Wegener's granulomatosis

\section{INTRODUCTION}

Wegener's granulomatosis (WG), first described in 1936, is a chronic systemic vasculitis that classically affects the upper respiratory tract, lower respiratory tract and kidneys. It affects small to medium sized blood vessels and causes necrotizing and granulomatous inflammation in the nose, paranasal sinuses, eyes, and lungs, necrotizing glomerulonephritits, and systemic involvement of many organ systems. ${ }^{1}$ It can manifest in two forms: limited disease without and generalized disease with renal involvement; most of the cases of generalized disease are associated with the presence of cytoplasmic anti-neutrophil cytoplasmic antibodies or C-ANCA. ${ }^{2}$

Owing to the systemic nature of the disease with a wide range of clinical presentations and the potential to involve any organ system in the body, patients often present to different specialists and the absence of a unifying hypothesis that explains all the features leads to piecemeal diagnosis delaying the already difficult diagnosis and appropriate treatment is often delayed. ${ }^{3}$ On the other hand, because of a low index of suspicion of WG due to its rarity, and confusion with other granulomatous diseases, particularly widely prevalent tuberculosis (TB), many patients are treated with antitubercular drugs causing a further delay in appropriate treatment. So it is not uncommon to see complications like irreversible organ damage like end stage renal disease by the time the disease is diagnosed. Here, we present two cases of WG, which were both histologically and serologically confirmed. Both of them offered many clues for diagnosis in earlier hospital visits, but it took two years for the first patient and eleven years for the second patient before a final diagnosis was made and appropriate therapy was started. 


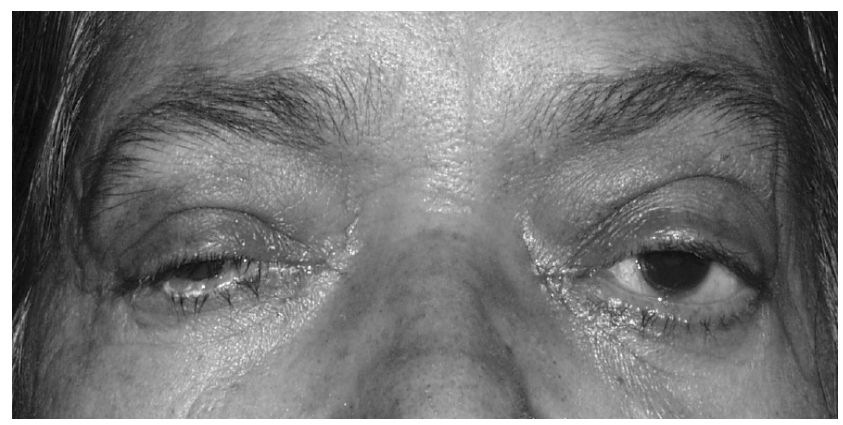

Figure 1. Bilateral corneal and scleral inflammation.

\section{CASE ONE}

A 61 year-old female presented to the Department of Ear, Nose, and Throat of a tertiary care hospital with a history of progressive throbbing headache and nasal bleeding of three months' duration. Examination revealed a left sided nasal mass and a CT scan of Paranasal Sinuses (PNS) showed a soft tissue lesion in the left nasal cavity and in all of the left sided PNS suggesting polyposis. Histopathological examination of the endoscopically debulked mass revealed dense inflammatory cells with multinucleate giant cells and the presence of neutrophilic inflammatory cells infiltrating the vascular wall with areas of necrosis. Anti-tubercular therapy (ATT) was started on the basis of histological finding of granulomatous inflammation.

However, her headache and nasal bleeding did not improve even after several weeks of ATT. Six weeks after the onset of nasal symptoms, the patient experienced painful red eyes. Ophthalmic evaluation revealed a bilateral scleritis and oral prednisolone was started. After a few weeks, she developed low grade fever, cough with blood streaked sputum, and joint pains. The patient was taken to a tertiary care hospital in a neighboring country where as an inpatient she developed mononeuritis multiplex in both feet, and a large necrotic ulcer at the site of intravenous canula in left forearm. As the patient's condition progressively deteriorated, she was asked to return back to Nepal. A repeat ophthalmic consultation in Kathmandu suggested bilateral ulcerative keratitis with anterior scleritis (Fig 1). USG of orbit suggested a pseudotumour within extraocular muscles. She was immediately referred to our hospital for further management.

At the time of admission in our hospital her BP was $150 / 90 \mathrm{mmHg}$; with normal pulse and temperature. She had bilateral ocular inflammation. A polypoid growth was visible in the left nasal cavity. There were multiple ulcers in the oral cavity, with another $3 \times 3 \mathrm{~cm}$ necrotic ulcer in the ventral aspect of the left forearm. The breath sounds were diminished in both lung bases. There was bilateral foot drop. Investigations revealed WBC 13,200/ $\mu \mathrm{L}$ (neutrophils $81 \%$, lymphocytes $13 \%$ ), Hb $11.1 \mathrm{~g} / \mathrm{dL}$, platelets $539000 / \mu \mathrm{L}$, and ESR $118 \mathrm{~mm}$ in 1st hour. The creatinine was $1.4 \mathrm{mg} /$ $\mathrm{dL}$ while the electrolytes and liver enzymes were normal. Chest $x$-ray showed multiple nodular opacities in the right lung with slightly enlarged cardiac silhouette. An

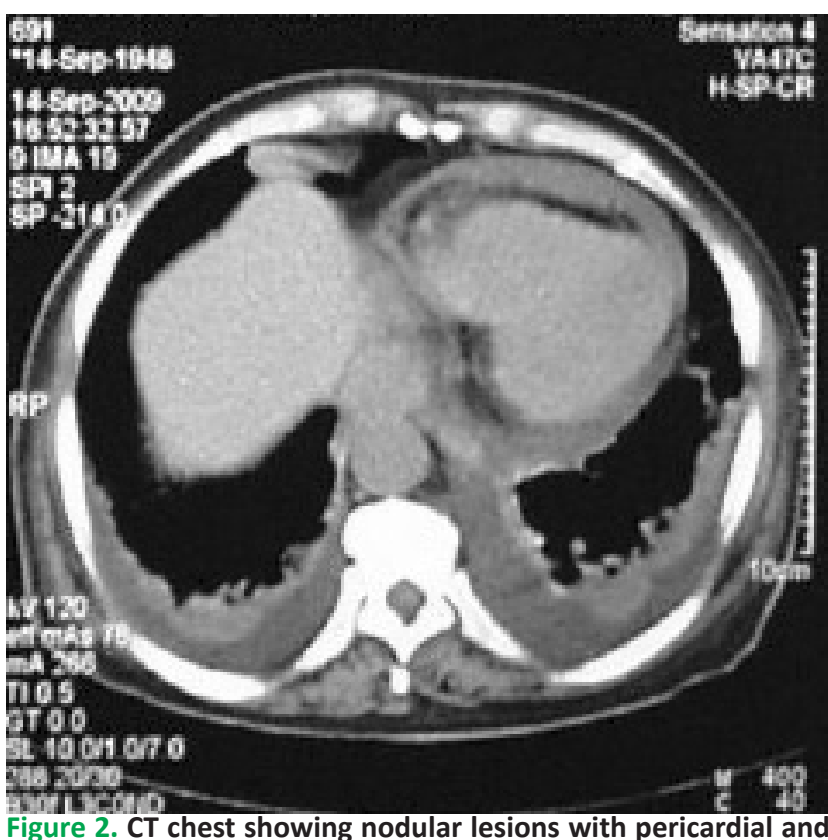

bilateral pleural effusions.

echocardiography revealed mild pericardial effusion. The Mantoux test revealed $15 \mathrm{~mm}$ induration after $72 \mathrm{hrs}$ of inoculation. Urine samples showed trace albumin, and persistence of microscopic haematuria. CT scan of the chest showed peripherally located irregular, non-enhancing nodules in both lungs with pleural and pericardial effusion (Fig 2).

MRI of lumbosacral spine showed mild degenerative changes in the lumbar spine without any compromise of the central canal and neural foramina.

Further investigations revealed negative serologies for hepatitis B, C, and HIV. The rheumatoid factor (RF) was positive and the anti-nuclear antibody (ANA) was negative. The c-ANCA was positive by indirect immunoflourescence method.

On the basis of multisystem nature of the disease, raised inflammatory markers, positive c-ANCA and biopsy findings, a diagnosis of generalized Wegener's granulomatosis was made. ATT was discontinued and Methylprednisolone pulse therapy was admisintered followed by tapering dose of steroids, and oral cyclophosphamide was instituted. The patient steadily improved and all of the features of active disease gradually disappeared. Now after 18 months of initiation of treatment, she enjoys a disease-free state maintained with low dose Azathioprine.

\section{CASE TWO}

A 38 year-old woman was referred to our hospital for the management of recently developed acute renal failure. The patient reported that she had a sudden onset of decreased urine output followed by progressive increasing swelling of body and shortness of breath. This followed a febrile illness 2 months ago. The renal function at the time of presentation was: urea $158 \mathrm{mg} / \mathrm{dL}$, creatinine $10.6 \mathrm{mg} / \mathrm{dL}$, 


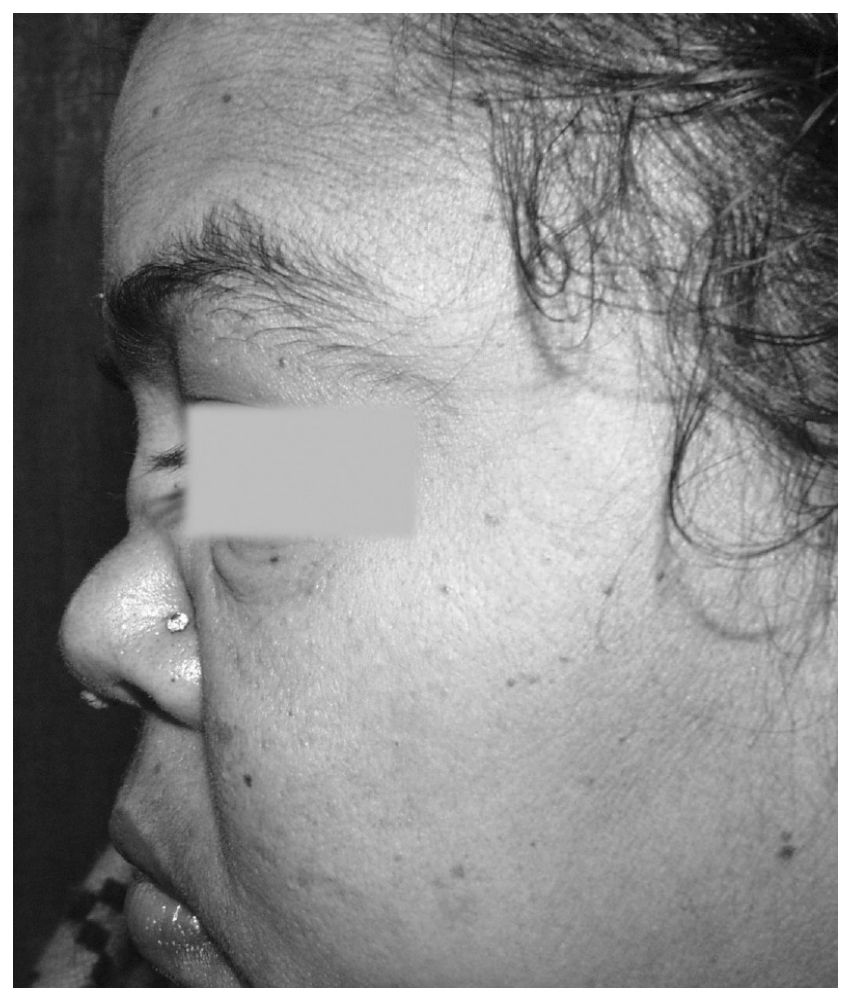

Figure 3. Saddle nose deformity due to nasal chondritis.

Sodium $135 \mathrm{mmol} / \mathrm{L}$, and Potassium 5.1mmol/L. The patient had already undergone a few sessions of hemodialysis in another centre before coming to our hospital.

Review of the past illness revealed that she had recurrent episodes of sinusitis when she was 27 years of age, and this was complicated to a saddle nose deformity several years ago (Fig 3). CT scan of the PNS revealed left maxillary antral mass with possible extension to inferior part of orbit (Figure 4).

The nasal septum was perforated. She had several biopsies from maxillary antral mass that showed fibrovascular tissue with areas of granulation and perivascular infiltrations of chronic inflammatory cells. The biopsies were inconclusive for infections and negative for malignant cells.

The patient had recurrent cough with hoarseness of voice six months before the current admission, bronchoscopy at that time showed edematous vocal cords. The patient also recalled that she had had reddish rashes in the lower extremities, and pain and swelling of the hand joints several months before this admission.

The most recent laboratory tests revealed WBC 10700/ $\mu \mathrm{L}$ (neutrophils 91\%, lymphocytes 6\%), hematocrit 26\%, platelets $281000 / \mu \mathrm{L}$, ESR $66 \mathrm{~mm}$ in Ist hour, normal liver function tests, urine showing proteinuria and microscopic haematuria. The HBsAg, HCV, HIV, and ANA were negative whereas c-ANCA (by indirect immunofluorescence) and RF were positive. On the basis of multisystem nature of the disease with positive serological and histological findings, WG was diagnosed. She was treated with 3 pulses of intravenous methylprednisolone followed by high dose prednisolone and oral cyclophosphamide along with 2

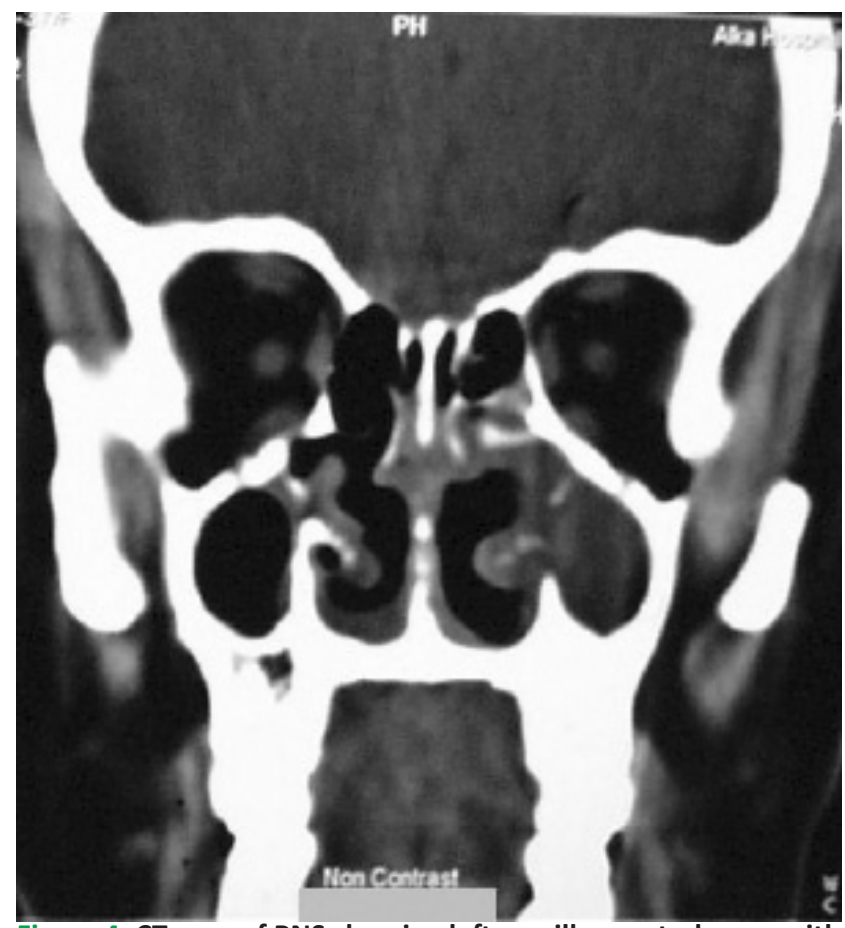

Figure 4. CT scan of PNS showing left maxillary antral mass with possible extension to left orbit.

sessions of maintenance haemodialysis every week. One month after the initiation of immunosuppressive treatment, she was much better with improvement in the systemic symptoms and tendency towards lesser requirements for haemodialysis.

\section{DISCUSSION}

WG is a rare disease, with the involvement of multiple organ systems; hence it is not uncommon for the affected patients to seek help from many physicians in many specialities. ${ }^{4}$ Majority of the patients with WG present with upper airway disease, occurring in more than $90 \%$ of cases. Common upper airway presentations include recurrent rhinosinusitis, nasal polyposis, nasal chondritis leading to saddle nose deformity, nasal septal perforation, serous otitis media, hearing impairment, and stridor due to subglottic stenosis. ${ }^{5}$ Lung involvement is one of the cardinal manifestations of Wegener's granulomatosis and is seen in up to $87 \%$ of patients. Common pulmonary manifestations include cough, haemoptysis, and shortness of breath with the underlying lesions as cavities, nodules, and fixed infiltrates. ${ }^{6}$ Renal involvement on kidney biopsy is present in up to $85 \%$ patients during the course of disease but in the majority the extra-renal disease often precedes the renal disease. The common renal manifestations are asymptomatic haematuria and proteinuria; however, it may progress to rapidly progressive glomerulonephritis (RPGN) with varying degrees of renal impairment as the disease becomes more severe. Immunohistologically, the glomeruli have no deposits of immunogloblins; hence 'pauciimmune necrotizing and crescentic glomerulonephritis' is sometimes used to describe this pattern. ${ }^{7}$ Eyes can be affected with epi/scleritis, retinal vasculitis and 
pseudotumour formation being common manifestations. Inflammatory arthritis, muscle pain, and variety of skin lesions ranging from palpable purpura to pyoderma gangrenosum are musculoskeletal manifestations of WG. Apart from these, any other organ systems could be affected by the vasculitic process, for example, nervous system, heart, gastrointestinal tract, etc. Constitutional symptoms like fever, weight loss, fatigue can be present at any time during the course of the active disease.

Both of our patients had presented with upper airway symptoms at the onset of the disease. As the disease progressed, these cases developed features of other organ involvement particularly eyes, skin, lungs, peripheral nerves, heart, and kidneys. Both the cases had prominent musculoskeletal symptoms during the course of disease. The first case developed prominent pulmonary involvement with lung nodules whereas the second case had a very severe renal disease (RPGN) requiring maintenance haemodialysis.

The serological marker of WG is the presence of c-ANCA. The c-ANCA pattern is seen by indirect immunofluorescence in a majority of active cases of WG (55\% with limited and $88 \%$ with systemic disease). ${ }^{8}$ These antibodies are directed specifically against proteinase - 3 (PR-3) in the azurophilic granules of neutrophils and the specificity of anti PR3 antibodies in the detection of WG is $99 \% .^{9}$ Both of our patients were positive for c-ANCA, meaning the disease process was active at the time of presentation at our centre.

Biopsy remains the gold standard for diagnosis of several types of vasculitis, particularly the small- to medium-vessel vasculitis like WG. Inflammatory lesions in WG typically include necrosis, granulomatous changes, and features of vasculitis. Though, vasculitis and granuloma formation can occur in the same lesion, it is unusual to see both in the same biopsy specimen. ${ }^{4}$ Moreover, renal biopsy in WG typically shows necrotizing inflammation without granuloma formation. Granulomatous inflammation, with features of vasculitis was present in both of our cases with features of necrosis as well in the first case. However, incorrect interpretation of the biopsy findings led to the delay in the initiation of specific treatment in both the cases.

WG and TB may present with similar clinical and histological findings; and one may confuse while taking care of such cases. ${ }^{10}$ Incorrect diagnosis of TB in a patient with WG may lead to the delay in institution of appropriate therapy with the development of resultant complications; on the other hand incorrect diagnosis of WG in a patient with TB leads to inappropriate treatment with immunosuppressive drugs, and this may even herald the development of military tuberculosis. ${ }^{11}$ However, with careful assessment of the progression of the disease, radiological characteristics, presence of c-ANCA and careful review of the results of the histological examination, it was established that these patients had WG.
These cases highlight the importance of considering WG in differential diagnosis of patients with multisystem disease. Close communication between physicians of different specialties is essential; at the same time an effective interaction between a clinician and a pathologist is equally important to avoid an erroneous diagnosis in the presence of equivocal biopsy test results.

\section{REFERENCES}

1. Khasnis A and Langford C. Update in Vasculitis. J Allergy Clin Immunol 2009; 123: 1227-38

2. Kallenberg CGM. Antineutrophil cytoplasmic autoantibody-associated small-vessel vasculitis. Curr Opin Rheumatol 2007; 19:17-24

3. D'Cruz DP, Baguley E, Asherson RA et al. Ear, nose, and throat symptoms in subacute Wegener's granulomatosis. Br Med J1989; 299:419-22

4. Bacon PA. The Spectrum of Wegener's Granulomatosis and Disease Relapse. New Engl J Med 2005, 352, 330-31

5. Murty GE. Wegener's Granulomatosis: Otorhhinolaryngological manifestations. Clin Otolaryngol 1990; 15: 385

6. Hoffman GS et al. Wegener's granulomatosis: An analysis of 158 patients. Ann Intern Med 1992; 116: 488-498

7. Jennete JC and Falk RJ. The pathology of vasculitis involving the kidney. Am J Kid Diseases 1994; 24:130-41

8. Gross WL, Schmitt WH, Csernok E. ANCA and associated diseases: Immunodiagnostic and pathogenetic aspects. Clin and Exp Immunol 1993;91:1-12

9. Hagen EC, Daha MR, Hermans J, et al. Diagnostic value of standardized assays for anticytoplasmic antibodies in idiopathic systemic vasculitis. Clin Nephrol 1998; 53: 743-53

10. Toyoshima M, Chiba K, Suda T et al. Wegener's Granulomatosis responding to Antituberculous drugs. Chest 2001; 119: 643-645

11. Harrison NK and Knight RK. Tuberculosis of the nasopharynx misdiagnosed as Wegener's granulomatosis. Thorax 1986; 41: 219220 